\title{
Endostar improved efficacy of concurrent chemoradiotherapy with vinorelbine plus carboplatin in locally advanced lung squamous cell carcinoma patients with high serum Lp(a) concentration
}

\author{
Hailing $\mathrm{Xu}^{1,2}$, Dongqing $\mathrm{Lv}^{2 \#}$, Yinnan Meng ${ }^{1,3}$, Miao Wang ${ }^{1,3}$, Wei Wang ${ }^{1,3}$, Chao Zhou ${ }^{1,3}$, Suna Zhou ${ }^{1,3}$, \\ Xiaofeng Chen ${ }^{4}$, Haihua Yang ${ }^{1,3 \#}$ \\ ${ }^{1}$ Laboratory of Cellular and Molecular Radiation Oncology, Radiation Oncology Institute of Enze Medical Health Academy, ${ }^{2}$ Department of \\ Pulmonary Medicine, Enze Hospital, ${ }^{3}$ Department of Radiation Oncology, ${ }^{4}$ Department of Cardiology, Affiliated Taizhou hospital of Wenzhou \\ Medical University, Taizhou 317000, China \\ Contributions: (I) Conception and design: H Yang, D Lv; (II) Administrative support: All authors; (III) Provision of study materials or patients: H \\ Yang, D Lv; (IV) Collection and assembly of data: H Xu, Y Meng, M Wang, W Wang, C Zhou, S Zhou; (V) Data analysis and interpretation: H Xu, \\ M Wang, W Wang, H Yang; (VI) Manuscript writing: All authors; (VII) Final approval of manuscript: All authors. \\ \#These authors contributed equally to this work. \\ Correspondence to: Haihua Yang, MD. Laboratory of Cellular and Molecular Radiation Oncology, Radiation Oncology Institute of Enze Medical \\ Health Academy, Department of Radiation Oncology, Affiliated Taizhou Hospital of Wenzhou Medical University, Taizhou 317000, China. \\ Email: yhh93181@hotmail.com; Dongqing Lv, MD. Department of Pulmonary Medicine, Enze Hospital, Affiliated Taizhou Hospital of Wenzhou \\ Medical University, Taizhou 317000, China. Email: lvdq@enzemed.com.
}

Background: The role of vascular targeting therapy combined with concurrent chemoradiotherapy (CRT) has produced many inconsistent results in locally advanced non-small-cell lung cancer (NSCLC), especially in lung squamous cell carcinoma (LSCC). Lipoprotein (a) $[\mathrm{Lp}(\mathrm{a})]$ may be critical in the development of tumor angiogenesis, and its levels are individualized and determined genetically. This study aimed to determine whether $\mathrm{Lp}(\mathrm{a})$ is correlated with effects of recombinant human endostatin (Endostar) combined with concurrent CRT for locally advanced LSCC.

Methods: Patients with locally advanced LSCC from December 2008 to December 2017 were retrospectively analyzed. Patients were divided into two groups: (I) a chemoradiotherapy group (CRT group) which received weekly vinorelbine and carboplatin concurrently with radiotherapy 60Gy, and (II) an Endostar in combination with chemoradiotherapy group (ECRT group) which received Endostar intravenous drip for 1-14 days (every 3 weeks) concurrently with CRT. Fasting venous blood samples for serum $\mathrm{Lp}(\mathrm{a})$ in all patients were collected before the treatment. The effect of Endostar was assessed by stratified analysis.

Results: A total of 94 patients were recruited in this study. There were 59 cases in the CRT group and 35 cases in the ECRT group. Overall, the median progression-free survival (PFS) was 9.6 vs. 14.2 months ( $\mathrm{P}=0.0671)$, and the overall survival (OS) was 15.0 vs. 20.6 months $(\mathrm{P}=0.114)$, in the CRT and ECRT groups respectively. The median of $\mathrm{Lp}(\mathrm{a})$ was $218 \mathrm{mg} / \mathrm{L}$. In patients with serum $\mathrm{Lp}$ (a) less than $218 \mathrm{mg} / \mathrm{L}$, the median PFS was 10.0 vs. 9.4 months $(\mathrm{P}=0.406)$, and the $\mathrm{OS}$ was 15.4 vs. 16.3 months $(\mathrm{P}=0.958)$ in the CRT and ECRT groups, respectively. However, in patients with serum $\mathrm{Lp}$ (a) higher than $218 \mathrm{mg} / \mathrm{L}$, the median PFS was 9.0 vs.15.8 months $(\mathrm{P}=0.011)$, and the OS was 14.0 vs. 21.1 months $(\mathrm{P}=0.055)$, in the CRT and ECRT groups, respectively. Cox proportional hazard model analysis revealed that a high concentration of $\mathrm{Lp}(\mathrm{a}), \geq 218 \mathrm{mg} / \mathrm{L}$, is a prognostic factor for PFS [hazard rate (HR), $0.43(0.23-0.81)]$ and OS [HR, 0.52 $(0.27-0.98)]$ in locally advanced LSCC $(\mathrm{P}<0.05)$.

Conclusions: The serum concentration of $\mathrm{Lp}(\mathrm{a})$ may serve as a biomarker to identify the patients who would benefit from Endostar treatment with concurrent CRT in stage III LSCC. 
Keywords: Recombinant human endostatin (Endostar); lipoprotein(a) [Lp(a)]; chemoradiotherapy (CRT); lung squamous cell carcinoma (LSCC)

Submitted Sep 29, 2019. Accepted for publication Jan 18, 2020.

doi: $10.21037 /$ apm.2020.01.16

View this article at: http://dx.doi.org/10.21037/apm.2020.01.16

\section{Introduction}

Lung cancer is the leading malignant disease in the world with high mortality and morbidity. Non-small-cell lung cancer (NSCLC) accounts for approximately $80 \%$ of the subtypes of lung cancer, among which lung squamous cell carcinoma (LSCC) accounts for about 30\%. Most LSCC patients are preliminarily diagnosed at an advanced stage with a mere $5-10 \%$ rate of 5 -year survival. With fewer driver gene mutations than adenocarcinoma and few new approaches to explore, there are only several therapies for inoperable advanced LSCC, including chemotherapy, chemoradiotherapy (CRT), and target therapy, resulting in an overall dissatisfactory survival time. Of late, CRT has been the first-line therapy for advanced LSCC patients showing 10-19 months of median progression-free survival (PFS) and 14-29 months of median overall survival (OS) $(1,2)$. However, treatment for CRT in locally advanced LSCC has plateaued, and so the modest benefit potentially offered by combination therapy attracted our attention.

Recombinant human endostatin (Endostar), a new antiangiogenic target drug, is efficient in blocking angiogenesis, inhibiting tumor endothelial cell proliferation, and suppressing primary tumor and metastatic growth in lung cancer with less risk of life-threatening side effects. Its underlying anti-tumor mechanisms are vascular endothelial growth factor (VEGF) signaling pathway, anti-apoptosis, and other processes $(3,4)$. It has been reported that Endostar combined with platinum-doublet chemotherapy, including vinorelbine, gemcitabine, paclitaxel, can prolong the survival time for those patients with good performance status in advanced LSCC, and has been recommended as the first-line choice, boasting 6.0-7.2 and 13.6-19.0 months of median PFS and OS, respectively $(5,6)$. However, the trend of improved survival time in advanced NSCLC patients administered maintenance treatment is still disputed $(7,8)$. Owing to its potential and promising benefits, emerging studies, including the latest HELPER study (9), have added insight into the effects of Endostar in combination with CRT in advanced NSCLC patients $(10,11)$. However, the superiority of Endostar combined with weekly vinorelbine plus carboplatin and radiation in locally advanced LSCC patients still needs further confirmation and exploring the prognostic factors for this regimen could offer urgently needed benefit.

Lipoprotein (a) $[\mathrm{Lp}(\mathrm{a})]$, composed of a low-density lipoprotein (LDL)-core with an apolipoprotein A [apo(A)] attached covalently to an apolipoprotein B-100 [apo(B-100)], has been found to be relevant for cancer and have cardiovascular potency (12), even though its antiangiogenic and antitumoral effects are still controversial and inconsistent. Marrer et al. (13) discovered no evident association between $\mathrm{Lp}(\mathrm{a})$ and cancer, whereas men with the highest $L p(a)$ levels seem to have the highest risk of lung cancer. Also, attributable to the increasing endothelial cell motility and promotion of angiogenesis through fibrin deposition, the concentration of $\mathrm{Lp}$ (a) has been found to be significantly elevated in lung cancer when compared with the normal population, and in stage IV patients when compared with the stage I group in breast cancer $(14,15)$. In addition to these, our previous study demonstrated an independently positive correlation between tumor stage and $\mathrm{Lp}$ (a) levels in lung cancer (16). Therefore, the increased value of $\mathrm{Lp}(\mathrm{a})$ in lung cancer and its underlying mechanisms should be emphasized in future studies.

Given this, our present study aimed to explore whether Endostar could improve the efficacy of concurrent CRT in locally advanced LSCC, and proposes a hypothesis that high $\mathrm{Lp}$ (a) concentration could be a prognostic factor for stage III LSCC patients in response to the regimen above.

\section{Methods}

\section{Patients and therapy}

This study retrospectively analyzed the locally advanced LSCC patients from December 2008 to December 2017. A total of 94 patients pathologically confirmed with locally advanced stage IIIA or IIIB LSCC were enrolled regardless of lines of treatment. The patients were divided into two 
groups: (I) the chemoradiotherapy group (CRT group) who received weekly vinorelbine $\left(12.5 \mathrm{mg} / \mathrm{m}^{2} / \mathrm{d}\right) /$ carboplatin [area under drug curve $(\mathrm{AUC})=2$ ] concurrently with radiotherapy consisting of 60 Gy total doses in 6 weeks, and (II) the Endostar in combination with chemoradiotherapy group (ECRT group) who received Endostar intravenous drip 1-14 days $\left(7.5 \mathrm{mg} / \mathrm{m}^{2} / \mathrm{d}, 3\right.$-week repetition program) on the basis of the CRT group. Additionally, relevant fasting venous blood samples including $\mathrm{Lp}(\mathrm{a})$ were collected before the treatment. This study was performed following an institutional ethics review board approved protocol.

\section{Evaluation criteria}

The primary endpoint was to explore a biomarker for the above setting regimen in LSCC patients while the second endpoints were PFS, OS, objective response rate (ORR) and adverse events (AEs). PFS was defined as the time to the patient's first clinical progression or death from any cause after concurrent CRT with or without Endostar. If there was no progression or the patient survived until our deadline was met, PFS was defined as the last date of confirmation of no progression. OS was defined as the time to death of any cause or most recent follow-up. According to response evaluation criteria in solid tumors (RECIST) version 1.1, the response to treatment could be clarified as complete response (CR), partial response (PR), stable disease (SD), and progressive disease (PD) at the first month after treatment. ORR was defined as the sum of the CR and PR percentages, while disease control rate (DCR) was defined as the sum of the of CR, PR, and SD percentages. AEs including nausea and vomiting, myelosuppression, radiation pneumonitis, radiation esophagitis, hypertension, and arrhythmia were recorded and classified into grades $0-5$.

\section{Statistical analysis}

All statistical procedures were performed on the statistical package for the social sciences (SPSS) version 20.0 (SPSS Inc., Chicago, IL, USA). Non-normal distributions were expressed as median and range, except for age, which was represented as the median and extreme. Comparisons between groups were performed by analysis of the nonparametric test for non-normally distributed variables as appropriate. The chi-square test was used for categorical variables. As for the PFS and OS, event-time distributions were estimated by the Kaplan-Meier method and the association between groups and survival was assessed by the log-rank test in the univariate analysis and stratified analysis. The Cox proportional hazards model was used subsequently to assess the contribution of each potential prognostic factor for survival. All $\mathrm{P}$ values were two-sided. A P value $\leq 0.05$ was considered statistically significant.

\section{Results}

\section{Patients' demographics and baseline characteristics}

The patients' characteristics at baseline are summarized in Table 1. A total of 94 LSCC patients were enrolled in this study. There were 59 cases in the CRT group, including 57 men and 2 women, while there were 35 cases in ECRT group, including 33 men and 2 women. In terms of age, sex, Karnofsky performance status (KPS), smoke status, and $\mathrm{Lp}(\mathrm{a})$, there was no difference of these baseline characteristics between the two groups according to chisquare analysis. The median $\mathrm{Lp}$ (a) was $218 \mathrm{mg} / \mathrm{L}$. On the basis of $\mathrm{Lp}(\mathrm{a})$, the stratification analysis was represented, and there was also no significant difference between groups.

Regarding the compliance of patients, the median follow-up time of patients was 20.25 months, while 26 of 59 and 22 of 35 patients completed the planned treatment schedule of concurrent CRT in the CRT and ECRT group, respectively. The ORR was achieved in $86.4 \%$ vs. $82.9 \%$ of patients in the CRT and ECRT group respectively. The DCR was $93.2 \%$ vs. $91.4 \%$ of patients in the CRT and ECRT group respectively. We observed the CR after treatment of the CRT group in 5 patients, $\mathrm{PR}$ in 46 patients, SD in 4 patients, and PD in 4 patients. After treatment in the ECRT group, there was PR in 29 patients, $\mathrm{SD}$ in 3 patients, and PD in 3 patients. The complete lack of CR in the experimental group might have been due to the limited number of patients (Table 2).

\section{Survival}

In the CRT and ECRT group respectively, the median PFS and $\mathrm{OS}$ were 9.6 vs. 14.2 months $(\mathrm{P}=0.0671)$ (Figure $1 A)$ and 15.0 vs. 20.6 months $(\mathrm{P}=0.114)$ (Figure $1 B)$.

In patients with less than $218 \mathrm{mg} / \mathrm{L}$, the median PFS and $\mathrm{OS}$ were 10.0 vs. 9.4 months $(\mathrm{P}=0.406)$, (Figure $2 A)$ and 15.4 vs. 16.3 months $(\mathrm{P}=0.958)$ (Figure $2 B)$ in the CRT and ECRT group, respectively. However, in patients with more than $218 \mathrm{mg} / \mathrm{L}$, the median PFS and OS were $9.0 v s .15 .8$ months $(\mathrm{P}=0.011)$ (Figure $2 C)$, and 14.0 vs. 21.1 months $(\mathrm{P}=0.055)$ (Figure $2 D)$ in the CRT and ECRT group 
Table 1 Patients' baseline characteristics $(\mathrm{N})$

\begin{tabular}{|c|c|c|c|c|c|c|c|}
\hline Characteristics & \multicolumn{3}{|c|}{ ECRT group } & \multicolumn{3}{|c|}{ CRT group } & $\mathrm{P}$ \\
\hline Age(years) & & & 0.69 & & & 0.35 & 0.45 \\
\hline$<62$ & 5 & 10 & & 15 & 15 & & \\
\hline$\geq 62$ & 8 & 12 & & 18 & 11 & & \\
\hline Male & 12 & 21 & & 31 & 26 & & \\
\hline Female & 1 & 1 & & 2 & 0 & & \\
\hline KPS & & & 0.065 & & & 0.87 & 0.33 \\
\hline 70 & 2 & 3 & & 4 & 9 & & \\
\hline Stage & & & 0.57 & & & 0.73 & 0.48 \\
\hline IIIA & 2 & 2 & & 15 & 13 & & \\
\hline IIIB & 11 & 20 & & 18 & 13 & & \\
\hline Smoke & & & 0.99 & & & 1.00 & 1.00 \\
\hline Non-smoke & 6 & 8 & & 4 & 4 & & \\
\hline Smoke & 7 & 14 & & 29 & 22 & & \\
\hline
\end{tabular}

CRT group, chemoradiotherapy group; ECRT group, Endostar in combination with chemoradiotherapy group.

Table 2 Response to treatment according to RECIST v.1.1

\begin{tabular}{lcccccc}
\hline Group & CR & PR & SD & PD & ORR & DCR \\
\hline ECRT group, $n(\%)$ & NA & $29(82.9)$ & $3(8.6)$ & $3(8.6)$ & $29(82.9)$ & $32(91.4)$ \\
CRT group, $n(\%)$ & $5(8.5)$ & $46(78.0)$ & $4(6.8)$ & $4(6.8)$ & $51(86.4)$ & $55(93.2)$ \\
\hline
\end{tabular}

CRT group, chemoradiotherapy group; ECRT group, Endostar in combination with chemoradiotherapy group; CR, complete response; PR, partial response; SD, stable disease; PD, progression disease; ORR, objective response rate; DCR, disease control rate; NA, not available.

respectively.

\section{Cox proportional hazard model analysis}

As is shown in the forest map analysis of PFS (Figure 3A), factors including age, sex, KPS, smoking status, stage, and $\mathrm{Lp}(\mathrm{a})$ were analyzed by Cox proportional hazards model. The results show that a high concentration of $\mathrm{Lp}$ (a) $(\geq 218 \mathrm{mg} / \mathrm{L})$ was a prognostic for patients with advanced LSCC [hazard rate (HR), 0.43(0.23-0.81)] $(\mathrm{P}<0.05)$. Meanwhile, similar outcomes for OS [HR, $0.52(0.27-0.98)]$ are displayed $(\mathrm{P}<0.05)$ in Figure $3 B$.
AEs

The AEs are outlined in Table 3. Overall, the most common grade $\geq 3 \mathrm{AE}$ was myelosuppression in both the CRT group (25.4\%) and ECRT group (37.1\%). Meanwhile, the number of patients with grade $\geq 3$ AEs was 19 (32.2\%) and 12 (34.3\%) in the CRT and ECRT group $(\mathrm{P}=0.658)$, respectively. For pneumonitis grade $\geq 3$ AEs, there were 3 patients $(5.1 \%) v s$. 1 patient $(2.9 \%)$ in the CRT and ECRT group, respectively. No grade $\geq 3$ adverse cardiovascular events including hypertension and arrhythmia were observed. There were no grade 5 events in either group. 

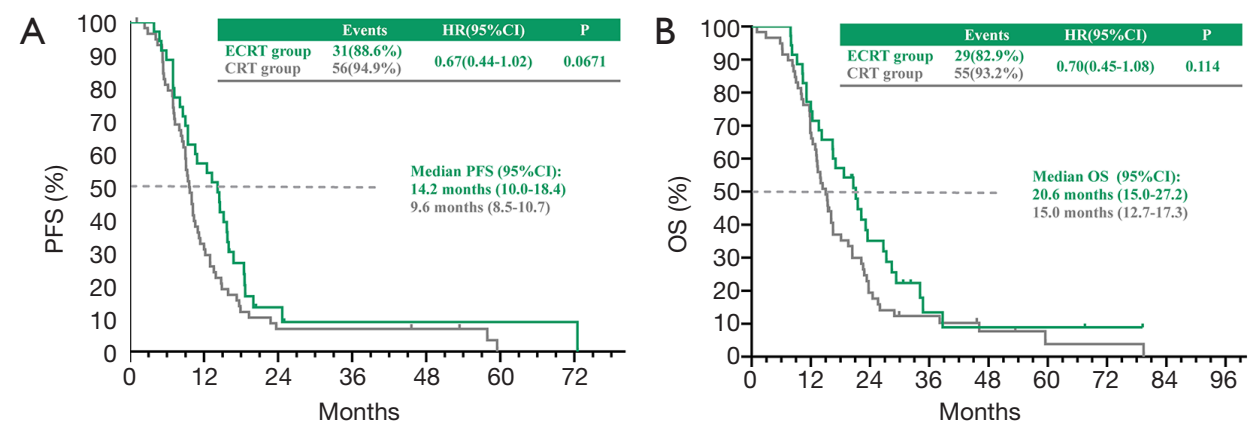

Figure 1 The PFS (A) and OS (B) in the CRT group and ECRT group. PFS, progression-free survival; OS, overall survival; CRT group, chemoradiotherapy group; ECRT group, Endostar in combination with chemoradiotherapy group; HR, hazard rate; CI, confidence interval.
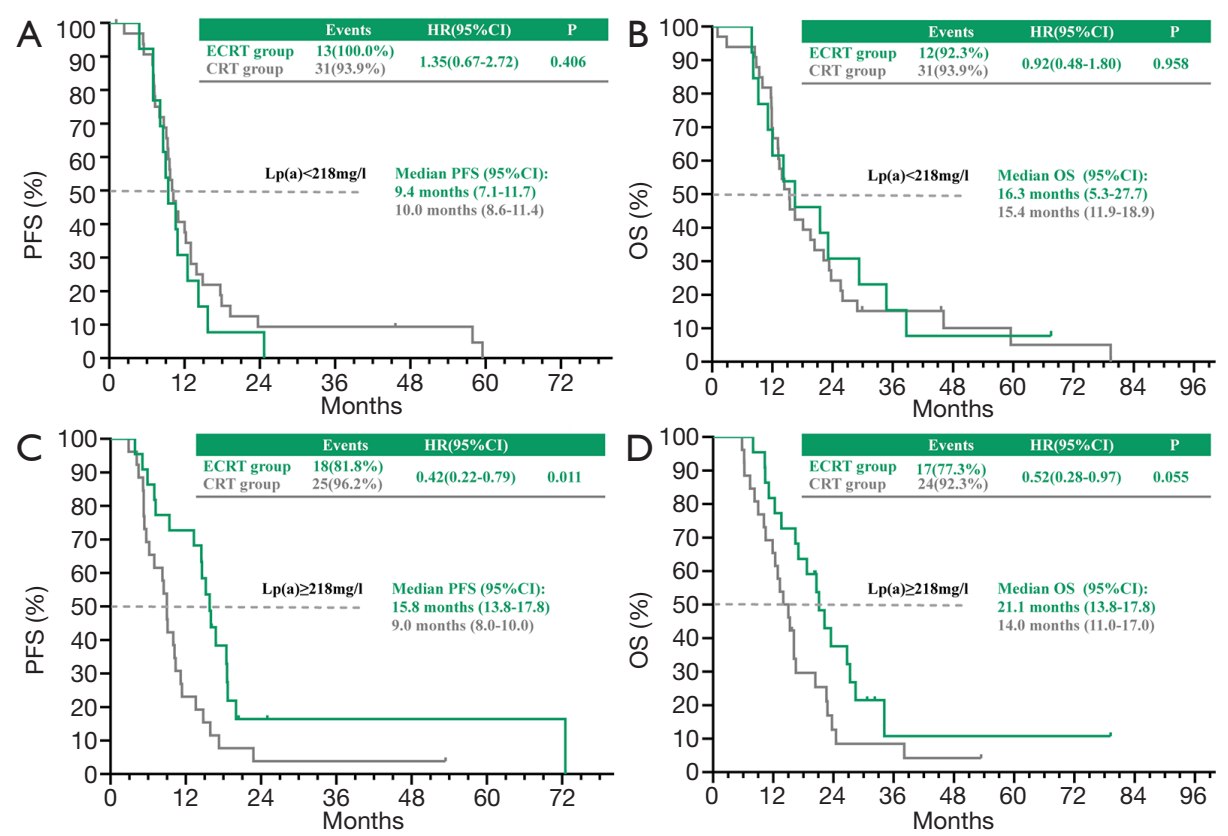

Figure 2 Stratified analysis of PFS (A) and OS (B) in the CRT group and ECRT group by low concentration of Lp(a); stratified analysis of PFS (C) and OS (D) in the CRT group and ECRT group by a high concentration of Lp(a). PFS, progression-free survival; OS, overall survival; Lp(a), lipoprotein(a); CRT group, chemoradiotherapy group; ECRT group, Endostar in combination with chemoradiotherapy group; HR, hazard rate; CI, confidence interval.

\section{Discussion}

This study proved that the regimen of concurrent radiation combined with vinorelbine plus cisplatin with the addition of Endostar can be tolerated in patients with locally advanced stage III LSCC. The concentration of Lp(a) may play a valuable role in identifying suitable patients for this regimen.

Patients with unresectable locally NSCLC have dissatisfactory survival times and poor quality of life, especially those with LSCC. Until now, CRT was recommended as the first line for stage III LSCC patients. As for the sequence of chemotherapy and radiotherapy, numerous studies have found that concurrent CRT could gain superior OS outcomes as a result of better locoregional control with mildly increased risk of esophageal toxicity $(17,18)$. It was observed that liposome-paclitaxel and carboplatin concurrent with radiotherapy showed a significant anti-tumor effect in LSCC patients with manageable toxicities, and had 17.0 months of estimated 
A

\begin{tabular}{lcc} 
Subgroup & \multicolumn{2}{c}{ ECRT } \\
All patients & $\mathbf{3 5}$ & $\mathbf{5 9}$ \\
Age, years & & \\
$<62$ & 15 & 30 \\
$\geq 62$ & 20 & 29 \\
Gender & & \\
male & 33 & 57 \\
*female & 2 & 2 \\
KPS & & \\
70 & 5 & 13 \\
80 & 23 & 37 \\
90 & 7 & 9 \\
Smoke & & \\
No & 4 & 8 \\
Yes & 31 & 51 \\
Stage & & \\
IIIA & 14 & 28 \\
IIIB & 21 & 31 \\
Lp(a), mg/l & & \\
$<218$ & 13 & 33 \\
$\geq 218$ & 22 & 26 \\
& &
\end{tabular}

B

Subgroup ECRT CRT

All patients $\quad 35 \quad 59$

Age, years

$\begin{array}{lll}<2 & 15 \quad 30\end{array}$

$\geq 62 \quad 20 \quad 29$

Gender

male $\quad 33 \quad 57$

*female 22

KPS

$\begin{array}{lll}70 & 5 & 13\end{array}$

$\begin{array}{lll}80 & 23 & 37\end{array}$

$90 \quad 7 \quad 9$

Smoke

No $4 \quad 8$

Yes $\quad 31 \quad 51$

Stage

IIIA $\quad 14 \quad 28$

IIIB $\quad 21 \quad 31$

Lp(a), mg/l

$<218$

$\geq 218$
PFS

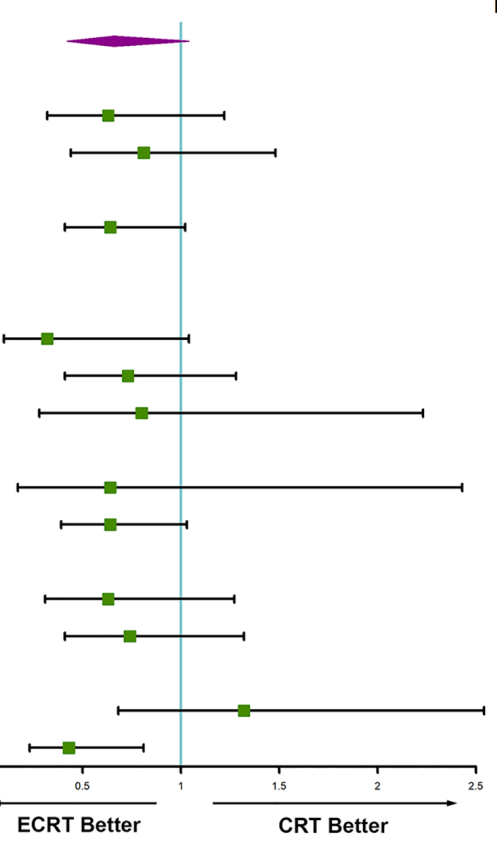

\section{Hazard Ratio(95\% $\mathrm{Cl})$ \\ $0.66(0.42-1.04)$}

$0.63(0.32-1.22)$

0.81(0.44-1.48)

0.64(0.41-1.02)

NA

$0.32(0.10-1.04)$

$0.73(0.41-1.28)$

$0.80(0.28-2.23)$

$0.64(0.17-2.43)$

0.64(0.39-1.03)

$0.63(0.31-1.27)$

0.74(0.41-1.32)

1.32(0.68-2.54)

$0.43(0.23-0.81)$

OS

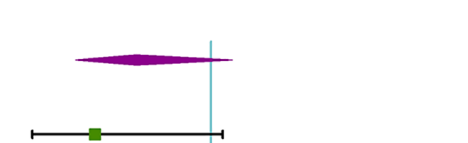

Hazard Ratio(95\% Cl)

0.69(0.44-1.09)

0.52(0.26-1.05)

$0.85(0.47-1.54)$

$0.67(0.42-1.06)$

NA

$0.66(0.21-2.07)$

$0.75(0.43-1.32)$

$0.58(0.17-1.92)$

$0.48(0.13-1.84)$

0.70(0.43-1.15)

$0.73(0.36-1.50)$

$0.69(0.38-1.26)$

$0.92(0.47-1.81)$

$0.52(0.27-0.98)$

Figure 3 Cox proportional hazardsmodel analysis on PFS (A) and OS (B) in the CRT group and ECRT group. *, analyzed data of females were not presented due to limited numbers. PFS, progression-free survival; OS, overall survival; CRT group, chemoradiotherapy group; ECRT group, Endostar in combination with chemoradiotherapy group; KPS, Karnofsky performance status; Lp(a), lipoprotein(a); HR, hazard rate; CI, confidence interval; NA, not available. 
Table 3 AEs

\begin{tabular}{|c|c|c|c|c|c|c|c|c|c|c|c|c|}
\hline Toxicity & \multicolumn{6}{|c|}{ CRT group (grade) } & \multicolumn{6}{|c|}{ ECRT group (grade) } \\
\hline Nausea/vomiting & 30 & 23 & 2 & 4 & 0 & 0 & 27 & 3 & 4 & 1 & 0 & 0 \\
\hline Myelosuppression & 4 & 21 & 19 & 14 & 1 & 0 & 1 & 11 & 10 & 12 & 1 & 0 \\
\hline Hypertension & 58 & 1 & 0 & 0 & 0 & 0 & 32 & 2 & 1 & 0 & 0 & 0 \\
\hline Esophagitis & 11 & 26 & 18 & 4 & 0 & 0 & 13 & 12 & 9 & 1 & 0 & 0 \\
\hline Pneumonitis & 16 & 27 & 13 & 3 & 0 & 0 & 16 & 14 & 4 & 1 & 0 & 0 \\
\hline
\end{tabular}

AEs, adverse events; CRT group, chemoradiotherapy group; ECRT group, Endostar in combination with chemoradiotherapy group.

median PFS, 29.0 months of median OS, and $68.4 \%$ of ORR (1). However, combination therapy was proven to be more efficient than CRT with improved survival time and similar treatment-toxicity.

Angiogenesis inhibitors, such as bevacizumab, cetuximab, Endostar etc., play a significant role in combination therapy for lung cancer patients. Random trials have demonstrated that the addition of bevacizumab to chemotherapy significantly improved the OS to 24.3 months in patients, regretfully with increased hemorrhage toxicity particularly in the squamous subtype (19). Cetuximab combined with vinorelbine plus cisplatin could obtain a statistically significant improvement in OS and better control rate for metastatic NSCLC, especially in a high expression ofepidermal growth factor receptor (EGFR) $(\geq 200)$ in the FLEX trial (20). The feasibility of cetuximab with CRT regiment was also demonstrated in radiation therapy oncology group (RTOG) 0324 with a 22.7 -month median OS (21). However, a negative outcome of cetuximab with CRT was observed with more AEs in RTOG 0617. Thus, whether cetuximab can offer a clear benefit of survival time in NSCLC patients, even with the high expression of EGFR, is still ambiguous.

The brand-new angiogenesis inhibitor, Endostar, has been shown to normalize vascular structure with reduced vessel diameter, increased pericyte coverage or proximity, and normalize basement membrane. It can also modulate functions of the tumor vascular network, by promoting oxygenation, reducing permeability, and improving the delivery efficiency of drugs, which are the basis of its antiangiogenic and anti-tumor effects with a low rate of AEs. There is a window phase after treatment with Endostar during which the tumor vascular network is normalized with good vascular structure and is then conducive to the exertion of a given drug's cytotoxic effects, contributing to increasing intra-tumor blood perfusion and improving the tumor hypoxia environment. This period begins on about the $4^{\text {th }}$ to the $10^{\text {th }}$ day after treatment of Endostar in vivo and vitro (22). Endostar was found to enhance radiosensitivity in Lewis lung carcinomas and clinical trials (23-25). After attempting to take advantage of this phenomenon, numerous studies demonstrated that Endostar in combination with CRT, whether it was paclitaxel, etoposide, or docetaxel, exerted a profound effect in stage III NSCLC patients $(11,26)$. Further to this, the gene delivery of Endostar into even a minority of tumor cells may be also an effective strategy to prevent progression of micrometastases to macroscopic disease (27). Consistent with previous results, we observed that in the CRT and ECRT group, the median PFS and OS were 9.6 vs. 14.2 months $(\mathrm{P}=0.067)$, and 15.0 vs. 20.6 months $(\mathrm{P}=0.114)$ respectively. This suggests that Endostar combined with CRT could modestly prolong PFS and OS and bring promising therapy for LSCC patients, although there was no statistically significant difference between the two groups.

In addition, grade $\geq 3 \mathrm{AEs}$ were observed in $32.2 \% v$ s. $34.3 \%$ of patients $(\mathrm{P}=0.658)$ in the CRT and ECRT group respectively. Pneumonitis AEs grade $\geq 3$ were $3 / 59$ (5.1\%) vs. $1 / 35$ (2.9\%) in the CRT and ECRT group respectively. Esophagitis grade $\geq 3$ AEs were 4/59 (6.8\%) vs. 1/35 (2.9\%) in the CRT and ECRT group respectively. There were no $\geq$ grade 3 cardiotoxicity events recorded and no grade 5 events. This indicates that the addition of Endostar into CRT would not add up toxicities for advanced LSCC patients with improved survival time. Even compared with some notable studies, Endostar in combination with weekly vinorelbine plus carboplatin may provide better security. Of course, it cannot be denied that the limited number 
of patients enrolled in our study, especially in the ECRT group, might have led to the low incidence of pneumonitis or esophagitis.

Interestingly, a meaningful association between the concentration of $\mathrm{Lp}(\mathrm{a})$ and the efficacy of LSCC in response to Endostar in combination with weekly vinorelbine plus carboplatin was observed. It was expected that there would be a higher concentration of $\mathrm{Lp}(\mathrm{a})$ in lung cancer compared with the normal population. It was also reported that there is a significant association between $\operatorname{Lp}(\mathrm{a})$ and the presence and stage of lung cancer. Some investigators have explained such effects may play a significant role in influencing cancer progression and extension rather than tumorigenesis. Moreover, the components of $\mathrm{Lp}(\mathrm{a})$ including $\mathrm{LDL}$ and apo(B-100) were shown to have a relationship with antiangiogenesis effects and the incidence of lung cancer. This is consistent with our results showing that high $\mathrm{Lp}(\mathrm{a})$ may be a favorable prognostic factor for locally advanced LSCC patients. We chose the median of $\mathrm{Lp}(\mathrm{a}), 218 \mathrm{mg} / \mathrm{L}$, as the cut-off. In patients with less than $218 \mathrm{mg} / \mathrm{L}$, the median PFS and OS were 10.0 vs. 9.4 months $(\mathrm{P}=0.406)$ (Figure $2 A)$, and 15.4 vs. 16.3 months $(\mathrm{P}=0.958)$ (Figure $2 B)$ in the CRT and ECRT group respectively. In patients with more than $218 \mathrm{mg} / \mathrm{L}$, the median PFS and OS were 9.0 vs.15.8 months $(\mathrm{P}=0.011)$ (Figure 2C), and 14.0 vs. 21.1 months $(\mathrm{P}=0.055)$ (Figure $2 D$ ) in the CRT and ECRT group respectively. However, the underlying mechanisms of $\mathrm{Lp}(\mathrm{a})$ in LSCC still need further exploration.

To our knowledge, the study was the first to evaluate the efficacy of an Endostar in combination with weekly vinorelbine plus carboplatin and radiation regimen for patients with LSCC, and to identify a common biology biomarker as a prognostic factor for LSCC patients. However, there were some limitations to the study. Firstly, it was a retrospective design rather than a prospective one, leading to a limited number of patients being in the ECRT group, although there was no difference in baseline characteristics. Also, the tolerance of consolidation therapy in patients with LSCC after treatment is thus far unknown. Furthermore, there were several female patients in our study. Several studies have revealed a close association between levels of estrogen and lipids (28), so that we could have either enrolled more female patients for the stratification analysis or excluded their statistics altogether.

In summary, we surmise that locally advanced LSCC patients with a high concentration of $L p(a)$ may benefit more from combined therapy with Endostar and concurrent CRT with weekly vinorelbine plus carboplatin. This marker may prove to be a valuable asset in treatment decision-making, patient selection, and the design of clinical trials.

\section{Conclusions}

For patients with locally advanced LSCC, our study demonstrated that the integration of Endostar and concurrent CRT with weekly vinorelbine plus carboplatin could be effective and safe, especially in a high $\mathrm{Lp}$ (a) concentration. The concentration of $\mathrm{Lp}(\mathrm{a})$ may be a prognostic factor for the Endostar combination with concurrent CRT in advanced stage III LSCC. We recommend the median Lp(a) in patients $(218 \mathrm{mg} / \mathrm{L})$ as the diacritical point. To be sure, these conclusions still should be prospectively validated and amended in a larger cohort study.

\section{Acknowledgments}

The authors gratefully acknowledge Dr. Feng-Ming (Spring) Kong, from Case Western Reserve University for editorial assistance of the abstract.

Funding: This work was supported in part by the Chinese National Science Foundation Projects (NSFC 81874221 and 81872458), the Zhejiang Provincial Medicine and Health Foundation (2015KYA240), and the Science and Technology Agency of Taizhou City (15yw01), China.

\section{Footnote}

Conflicts of Interest: The authors have no conflicts of interest to declare.

Ethical Statement: The authors are accountable for all aspects of the work in ensuring that questions related to the accuracy or integrity of any part of the work are appropriately investigated and resolved. This study was performed following an institutional ethics review board approved protocol (K20130227).

Open Access Statement: This is an Open Access article distributed in accordance with the Creative Commons Attribution-NonCommercial-NoDerivs 4.0 International License (CC BY-NC-ND 4.0), which permits the noncommercial replication and distribution of the article with the strict proviso that no changes or edits are made and the original work is properly cited (including links to both the formal publication through the relevant DOI and the license). 
See: https://creativecommons.org/licenses/by-nc-nd/4.0/.

\section{References}

1. Chen G, Sheng L, Du X. Efficacy and safety of liposome-paclitaxel and carboplatin based concurrent chemoradiotherapy for locally advanced lung squamous cell carcinoma. Cancer Chemother Pharmacol 2018;82:505-10.

2. Lancia A, Merizzoli E, Filippi AR. The 8th UICC/AJCC TNM edition for non-small cell lung cancer staging: getting off to a flying start? Ann Transl Med 2019;7:S205.

3. Ling Y, Yang Y, Lu N, et al. Endostar, a novel recombinant human endostatin, exerts antiangiogenic effect via blocking VEGF-induced tyrosine phosphorylation of KDR/Flk1 of endothelial cells. Biochem Biophys Res Commun 2007;361:79-84.

4. Massabeau C, Rouquette I, Lauwers-Cances V, et al. Basic fibroblast growth factor-2/beta3 integrin expression profile: signature of local progression after chemoradiotherapy for patients with locally advanced non-small-cell lung cancer. Int J Radiat Oncol Biol Phys 2009;75:696-702.

5. Xing $\mathrm{P}, \mathrm{Hao} \mathrm{X}, \mathrm{Hu} \mathrm{X}$, et al. Recombinant Human Endostatin in the Treatment of Advanced Lung Squamous Cell Carcinoma. Zhongguo Fei Ai Za Zhi 2016;19:670-4.

6. Sun Y, Wang JW, Liu YY, et al. Long-term results of a randomized, double-blind, and placebo-controlled phase III trial: Endostar (rh-endostatin) versus placebo in combination with vinorelbine and cisplatin in advanced non-small cell lung cancer. Thorac Cancer 2013;4:440-8.

7. Zhou S, Zuo L, He X, et al. Efficacy and safety of rhendostatin (Endostar) combined with pemetrexed/ cisplatin followed by rh-endostatin plus pemetrexed maintenance in non-small cell lung cancer: A retrospective comparison with standard chemotherapy. Thorac Cancer 2018;9:1354-60.

8. Senan S, Brade A, Wang LH, et al. PROCLAIM: Randomized Phase III Trial of Pemetrexed-Cisplatin or Etoposide-Cisplatin Plus Thoracic Radiation Therapy Followed by Consolidation Chemotherapy in Locally Advanced Nonsquamous Non-Small-Cell Lung Cancer. J Clin Oncol 2016;34:953-62.

9. Zhai Y, Ma H, Hui Z, et al. HELPER study: A phase II trial of continuous infusion of endostar combined with concurrent etoposide plus cisplatin and radiotherapy for treatment of unresectable stage III non-small-cell lung cancer. Radiotherapy and Oncology 2019;131:27-34.

10. Tang H, Ma H, Peng F, et al. Prognostic performance of inflammation-based prognostic indices in locally advanced non-small-lung cancer treated with endostar and concurrent chemoradiotherapy. Mol Clin Oncol 2016;4:801-6.

11. Bao Y, Peng F, Zhou QC, et al. Phase II trial of recombinant human endostatin in combination with concurrent chemoradiotherapy in patients with stage III non-small-cell lung cancer. Radiother Oncol 2015;114:161-6.

12. Lippi G, Franchini M, Salvagno GL, et al. Lipoprotein[a] and cancer: anti-neoplastic effect besides its cardiovascular potency. Cancer Treat Rev 2007;33:427-36.

13. Marrer E, Wagner A, Montaye M, et al. Lipoprotein(a) plasma levels and the risk of cancer: the PRIME study. Eur J Cancer Prev 2013;22:286-93.

14. Lyu Z, Li N, Wang G, et al. Independent and joint associations of blood lipids and lipoproteins with lung cancer risk in Chinese males: A prospective cohort study. Int J Cancer 2019;144:2972-84.

15. Van Wersch JW. The behaviour of lipoprotein(a) in patients with various diseases. Scand J Clin Lab Invest 1994;54:559-62.

16. Yang HH, Chen XF, Hu W, et al. Lipoprotein(a) level and its association with tumor stage in male patients with primary lung cancer. Clin Chem Lab Med 2009;47:452-7.

17. Curran WJ, Jr., Paulus R, Langer CJ, et al. Sequential vs. concurrent chemoradiation for stage III non-small cell lung cancer: randomized phase III trial RTOG 9410. J Natl Cancer Inst 2011;103:1452-60.

18. Fournel P, Robinet G, Thomas P, et al. Randomized phase III trial of sequential chemoradiotherapy compared with concurrent chemoradiotherapy in locally advanced non-small-cell lung cancer: Groupe Lyon-SaintEtienne d'Oncologie Thoracique-Groupe Francais de Pneumo-Cancerologie NPC 95-01 Study. J Clin Oncol 2005;23:5910-7.

19. Wozniak AJ, Moon J, Thomas CR Jr, et al. A Pilot Trial of Cisplatin/Etoposide/Radiotherapy Followed by Consolidation Docetaxel and the Combination of Bevacizumab (NSC-704865) in Patients With Inoperable Locally Advanced Stage III Non-Small-Cell Lung Cancer: SWOG S0533. Clin Lung Cancer 2015;16:340-7.

20. Pirker R, Pereira JR, Szczesna A, et al. Cetuximab plus chemotherapy in patients with advanced non-small-cell lung cancer (FLEX): an open-label randomised phase III trial. Lancet 2009;373:1525-31.

21. Nieder C. RTOG study 0324: Cetuximab in combination with chemoradiation in patients with stage IIIA/B non- 
small-cell lung cancer. Strahlenther Onkol 2012;188:194-5.

22. Lv Y, Jiang R, Ma C, et al. Clinical Observation of Recombinant Human Vascular Endostatin Durative Transfusion Combined with Window Period Arterial Infusion Chemotherapy in the Treatment of Advanced Lung Squamous Carcinoma. Zhongguo Fei Ai Za Zhi 2015;18:500-4.

23. Luo X, Slater JM, Gridley DS. Enhancement of radiation effects by pXLG-mEndo in a lung carcinoma model. Int J Radiat Oncol Biol Phys 2005;63:553-64.

24. Meng MB, Jiang XD, Deng L, et al. Enhanced radioresponse with a novel recombinant human endostatin protein via tumor vasculature remodeling: experimental and clinical evidence. Radiother Oncol 2013;106:130-7.

25. Jiang XD, Dai P, Wu J, et al. Effect of recombinant

Cite this article as: $\mathrm{Xu} \mathrm{H}, \mathrm{Lv} \mathrm{D}$, Meng Y, Wang M, Wang W, Zhou C, Zhou S, Chen X, Yang H. Endostar improved efficacy of concurrent chemoradiotherapy with vinorelbine plus carboplatin in locally advanced lung squamous cell carcinoma patients with high serum $\mathrm{Lp}$ (a) concentration. Ann Palliat Med 2020;9(2):298-307. doi: 10.21037/apm.2020.01.16 human endostatin on radiosensitivity in patients with non-small-cell lung cancer. Int J Radiat Oncol Biol Phys 2012;83:1272-7.

26. Sun XJ, Deng QH, Yu XM, et al. A phase II study of Endostatin in combination with paclitaxel, carboplatin, and radiotherapy in patients with unresectable locally advanced non-small cell lung cancer. BMC Cancer 2016;16:266.

27. Folkman J. Antiangiogenesis in cancer therapy-endostatin and its mechanisms of action. Exp Cell Res 2006;312:594-607.

28. Kim CJ, Ryu WS, Kwak JW, et al. Changes in Lp(a) lipoprotein and lipid levels after cessation of female sex hormone production and estrogen replacement therapy. Arch Intern Med 1996;156:500-4. 